\title{
A GENERALIZED MULTIPLE RESPONSE SURFACE METHODOLOGY FOR COMPLEX COMPUTER SIMULATION APPLICATIONS
}

\author{
Jeffrey B. Schamburg \\ Operations Research Center \\ Department of Systems and Information Engineering \\ United States Military Academy \\ West Point, NY 10996, U.S.A.
}

\author{
Donald E. Brown \\ Department of Systems and Information Engineering \\ University of Virginia \\ Charlottesville, VA 22901, U.S.A.
}

\begin{abstract}
This work provides a generalization of the traditional response surface methodology (RSM) that can be applied to complex, multi-objective simulation studies. These problems involve a larger number of input variables, multiple measures of performance, and complex systems relationships. This multiple RSM approach capitalizes on the underlying learning philosophy of the traditional RSM while benefiting from other knowledge discovery concepts and data mining techniques. Furthermore it does not require the restrictive assumptions of the traditional RSM nor does it restrict the analyst to the traditional RSM techniques. Based on a variation of (Brown and Schamburg 2004) and (Schamburg 2004), a brief description of the generalized approach is provided. Then, the multiple response techniques are shown through an example application.
\end{abstract}

\section{INTRODUCTION}

The purpose of this paper is to provide an improved methodology that allows analysts to learn from the use of complex multiple response computer simulations. Computer simulations are becoming more popular and are being used in a variety of domains to include production, transportation, economics, biology, ecology, law enforcement, military operations, behavioral and social sciences and others. As computer capabilities have increased, the complexity of many simulations has increased as well. However, the capabilities of complex simulations create the following issues for analysts:

1. A need for human learning through the analysis of complex simulations

2. Analysis involving a large number of variables, rules, multiple measures of performance, and complex relationships

3. Analysis involving a broad decision space
4. Finding good solutions and determining tradeoffs among many factors and different types of factors

5. Understanding complex relationships

6. Developing generalizations and providing implications for decision making and operational procedures

For analysis, often times the systems engineer is forced to make numerous simplifying assumptions and then select a few variables over a limited domain. In other cases, trial and error or heuristic optimization techniques are used. These methods are not well suited for human learning and do not take advantage of the discovery potential of such large scale simulations. This work addresses this need through the development of a general approach to improve analysis methods for complex multiple response computer simulations.

\section{THE TRADITIONAL RESPONSE SURFACE METHODOLOGY}

The traditional response surface methodology (TRSM) provides an approach for human learning through analysis. Through the use of TRSM, human analysts gain knowledge with an iterative process involving the steps, "conjecture, design, experiment, and analysis, (Box and Draper 1987)." In developing empirical models, the analyst must first "conjecture" as to the form of the model which may be used to represent the system over a given portion of the solution space. With TRSM, first order and second order linear regression models are normally conjectured. The analyst then "designs" a "suitable experiment to test, estimate, and develop the current conjectured model." Common RSM experimental designs include factorial, central composite, and Box-Behnken experimental designs. Next, he conducts the "experiment." Using the resulting data from the experiment, he then conducts the "analysis." The analysis involves the use of t-statistics, analysis of variance, and graphical analysis techniques. 
TRSM has been predominantly used in the analysis of physical systems found in manufacturing, production, and chemical processing. However, it has also been used in the analysis of computer simulations but these applications are usually limited in the complexity, domain, and the number of variables in the analysis. Nonetheless, the TRSM offers advantageous concepts for human learning. This process of learning (through the use of experimentation, model development, and analysis) provides the basis for our approach that was developed to address the issues previously brought forth in the introduction.

\section{THE GENERALIZED MULTIPLE RESPONSE SURFACE METHODOLOGY}

Using the fundamental learning concepts of the TRSM as a starting point, the generalized multiple RSM incorporates other data mining techniques and new experimental design concepts for the purpose of knowledge discovery. This generalized approach is a modification of (Schamburg 2004). Compared to the TRSM, this generalized methodology can be applied to more complex systems engineering problems. These problems have the following characteristics: a larger decision space with more factors of interest; multiple measures of performance; and complex systems relationships. Furthermore, this generalized multiple RSM is intended for problems where: 1) the analyst begins the analysis with relatively little understanding of the variable relationships in the systems under study; or 2) the analyst is interested in understanding a larger decision space without having to select a limited number of variables for analysis. These analysis problems may have multiple purposes. These may include: 1) determining near optimal solutions; 2) understanding the tradeoffs among important variables in the study; and 3) translating the findings into generalizations for operational procedures. Through multiple data mining approaches and by allowing the analyst to look over a larger decision space with more variables, this generalized multiple RSM better addresses these problem situations and purposes.

In an iterative way, this developed methodology provides a process for learning through experimentation and analysis. The initial phase of the methodology is identified as a "search" analysis. That is, the analysis begins with an investigation of a large decision space with many variables. As necessary, later iteration(s) of the methodology may be more focused. The following provides the 8 steps of the general framework for the generalized multiple RSM. The developed methodology is a general one and may be adjusted to meet the needs of the analysis application, (Schamburg 1995), (Brown and Schamburg 2004), and (Schamburg 2004). For more detail see (Brown and Schamburg 2004) and (Schamburg 2004).

Step 1. Define the Analysis. Defining the analysis consist of the three following parts.
Determine the Issues for Analysis. The analysis begins by determining the issues and concepts upon which the study will focus.

Define and Prioritize Performance Metrics. In this step we define the measures of system performance and prioritize them. In the next section of this paper, we provide techniques, through example, for dealing with multiple responses in a robust way.

Develop the Scenario. Here we determine the environment and the hierarchical level of the system that we want to investigate.

Step 2. Prepare the Simulation. Preparing the simulation consists of developing the simulation representation of the problem and calibration.

Develop the Simulation Representation of the Problem. Here we determine specific parameter values for the subsystems in the study. If possible, reliable, external data is used to help determine parameter values where necessary. In addition, subject matter input can be used.

Calibrate the Simulation Model. Once the simulation is set up, we run the simulation and compare the outputs to trusted or live data if available. Based on the results, the simulation parameters can be scaled and then adjusted so that the output is similar to the live data.

Step 3. Conjecture. Here we determine the data mining approach or the order of the model required in the analysis. This step requires that we conjecture as to the form of the model which may be used to represent the system over a given portion of the solution space. Appropriately determining the order (or type) of the model in this step leads to an appropriate set of experiments in the next step. Different data mining approaches may have different training data needs.

For the initial iteration of this methodology, we typically look over a broad portion of the solution space and expect higher order models to be appropriate. For the step that follows, these models require experimental designs with many levels. This is especially true during the initial iteration of the methodology. We may conjecture, for example, that higher order models, developed through least squares, may be appropriate. On the other hand, logistic regression, classification and regression trees (CART's), neural networks, or other types of models may be appropriate.

As the methodology progresses and the analysis region is reduced, simpler models may be conjectured. In some cases for example, the analysis may lead one to conjecture that first order models in which two or three level factorial designs may be appropriate for the next step.

For comparison, consider the common progression in a TRSM analysis. In the initial phases of the TRSM, first order models are usually appropriate. It may be believed that little curvature is expected until the experimentation is nearer to the optimum. However, when curvature is encountered in previous phases or expected in the upcoming experimentation, a higher order model should be conjectured. Although various transformations may be attempted to limit the order of the model, strong curvature and interactions typically necessitate the use of higher order models. 
Step 4. Design. Here the analyst selects an experimental design. This step of the methodology includes determining which variables and what levels of these variables should be considered for an analysis. In part, this is directed by the issues for analysis.

Through the use of Latin hypercube designs, the generalized multiple RSM is appropriate for a large number of variables. Because of the space-filling properties and the larger number of variables that they can accommodate, we have used Cioppa's nearly orthogonal Latin hypercube designs in our applications with this methodology, (Cioppa 2002). Cioppa's designs allow us to look at up to 22 variables at 129 levels. While maintaining "near orthogonality," these designs also have good space-filling properties, allowing us to get a better representation of the entire decision space. Because they are nearly orthogonal and because they allow up to 129 levels for continuous variables, these designs allow us to get a reasonable understanding of the variable relationships with the response(s) over a broader range.

In some cases, a modification of the Latin hypercube designs is necessary when one or more variables have a fewer number of discrete levels or when one or more variables are categorical. In these cases, one approach is to round the design specified level to the nearest discrete level allowed for the variable. If this approach is used, the analyst should look at the pairwise correlation within the experimental design to make sure the design is still orthogonal (or nearly orthogonal). Another approach is to put together several Latin hypercube designs so that each individual Latin hypercube accounts for a different level or a different category of the noncontinuous variable within the overall design.

For some iterations of the methodology, primarily in later iterations, the common TRSM experimental designs may be appropriate. These may include factorial, fractional factorial, central composite, and Box-Behnken experimental designs. However, these designs limit the number of variables and the number of levels in the analysis.

Step 5. Experiment. Here the analyst conducts the designed set of experiments through use of the developed computer simulation program. The experiments yield the measures of performance.

Step 6. Conduct the Analysis. This step consists of the following four parts.

Conduct an Exploratory Data Analysis. The exploratory data analysis is used to determine which factors and interactions are most important and how they affect the response(s). Basic descriptive statistics and graphical techniques are used to conduct the exploratory data analysis. Descriptive statistics and histograms, for example, may be used to check the distributions of the responses. When appropriate, this may be helpful in selecting transformations when training the models in the next step of the methodology. As another example, each response may be graphed against each input factor to help determine with factors might be most important in the analysis. The exploratory data analysis, in part, addresses some of the issues and concerns brought forth at the beginning of the process. It also helps determine which terms may be most important in training the models.

Train the Models. This step requires the determination and fitting of appropriate mathematical models from which to analyze the relationships between the input variables and the response variables. Separate models are developed for each response. For analysis of the relationships, the use of classification and regression tree (CART) techniques and empirical model development through regression are complementary in the following steps of the methodology. In some applications, nearest neighbor techniques, neural networks, support vector machines or other modeling approaches may be appropriate. Our multiple response approach, described in the next section, requires the development of multiple classification and regression trees (CART's).

To help further understand the complex relationships over broad experimental regions, higher order function approximations developed through least squares or logistic regression may be useful. These function approximations may include restricted cubic splines (RCS's) in addition to first order, second order, and interaction terms.

Models with RCS terms can be more flexible than simple second order polynomials and can be used to better represent non-linear relationships while providing models that are linear in the tails of the relationships. An empirical model with RCS's is formed by dividing the ranges of the factors into intervals and developing a piecewise function. The endpoints of the intervals are called knots. As an example, a function with one factor $X$ and $j$ knots would have $j+2$ terms, counting the intercept. These functions have the form:

$$
\begin{aligned}
f(X)= & \beta_{0}+\beta_{1} X+\beta_{2}\left(X-k_{1}\right)_{+}^{3}+ \\
& \beta_{3}\left(X-k_{2}\right)_{+}^{3} \ldots+\beta_{j+1}\left(X-k_{j}\right)_{+}^{3}
\end{aligned}
$$

where the $\beta_{i}$ are the estimated coefficients,

$$
(z)_{+}=\left\{\begin{array}{clc}
z, & \text { if } & z>0 \\
0 & \text { if } & z \leq 0
\end{array},\right.
$$

and the $k_{j}$ represent the knots. Because the RCS's are forced to be linear in the tails, only $\mathrm{j}$ coefficients, counting the intercept, have to be calculated. The remaining 2 coefficients are found as functions of the other terms in the model. Normally, $\mathrm{j}=3$, 4, or 5. See (Harrell 2001). (Stone and Koo 1985) and (Harrell 2001) describe other concepts and advantages of RCS's.

Judge the Adequacy of Fit of the Models. The models can be judged through use of statistical analysis, analysis of the mean square error, residual analysis techniques, or through the performance on test data. If a model fails particular tests, we may attempt to try a different transformation of the data or a different data mining approach and 
then return conduct this step again. If a model does not satisfactorily predict the response, return to step 4 above, make adjustments to the experiment and go through the sequence again to improve the model.

Determine Tradeoffs and Analyze Relationships. Of the factors considered, we determine which of these factors or combinations of factors have the greatest effect on the responses. The sensitivity analysis includes an analysis of the tradeoffs among these factors and the responses. To accomplish this we review the exploratory data analysis, the resulting CART, and the statistics from the developed function approximations. For this purpose, analyses through use of the developed function approximations and CART are complementary.

Analysis of a graphical representation of the empirical models and the CART is one of the final procedures in this methodology. A graphical representation of the response surface may be provided through the use of contour diagrams and response surface plots. Analysis of these graphs shows the relationship between two variables and the response when the values of all other variables are held constant. The contour diagram is a two dimensional representation of constant response contours over the ranges of values for two variables. The response surface plot is a three dimensional representation of the response surface over the ranges of values for two variables. For any pair of variables, multiple graphs may be analyzed by changing the values of the other variables. These graphical representations of the response surface allow one to investigate and analyze the interactions, relationships, and tradeoffs between a response variable and pairs of input variables. As we go through this process for each of the response variables, we begin to understand how improvement (or degradation) in one area might cause degradation (or improvement) in another area.

Step 7. Determine Near Optimal Settings and Constrain the Decision Space. This step is accomplished by using the special structure of the developed CART's. This multiple response approach uses the CART's in an iterative way, based on prioritization of the responses, in order to determine near optimal settings and constrain the decision space. This information is used to help determine the next experimental region in the next iteration of the methodology. Alternative approaches to this step may consider the use of goal programming or heuristic search techniques considering decision analysis concepts.

Step 8. Develop Generalizations and Address the Issues for Analysis. Through the analysis and conclusions found in the steps above, we attempt to make generalizations that will be beneficial in developing procedures or in decision making related to the system under study. In this step we attempt to address the key issues and summarize the most important findings in our analysis.

\section{THE MULTIPLE RESPONSE SURFACE TECHNIQUE}

This section describes aspects of the multiple RSM technique that is used with the generalized RSM approach described in the previous section. These concepts further define the second part of step 1 and step 7 above. This method involves the development and use of CART's for each response. Through an iterative sequence of the steps described above, the CART's are used, one-by-one, based on the prioritization of the responses. As the individual CART's are used, the decision space is constrained and near optimal solutions are determined. This multiple RSM technique is shown through use of a small infantry unit attack scenario.

This small infantry unit attack analysis involves the investigation of information technologies for infantry soldiers. In this application we are interested in how these technologies might affect procedures at the fire team and squad levels. The squad consists of a squad leader plus two fire teams with four soldiers each. In total there are nine soldiers in the friendly squad.

The potential future technologies considered in this analysis include: an unmanned aerial sensor, squad situational awareness software, squad call for fire capabilities, and squad communications. With the squad call for fire capabilities, the squad can request support from company mortars. Also, the squad leader has the ability to request fire support from higher echelon non-line of sight fires. We vary the capabilities of these technologies as part of the analysis.

The friendly mission is to destroy the enemy force and to seize a key position. The enemy force is an enemy platoon defending from a fortified position. The platoon consists of a platoon leader and three infantry squads. There are a total of 39 enemy solders in this scenario. The enemy force has advantages of survivability as a result of being in this defensive position. Furthermore, for early warning and use of indirect fire, the enemy has listening and observation posts that are forward of the defended position. Normally, friendly forces desire a 3 to 1 advantage for offensive operations of this nature. However, for this analysis, we developed the enemy force to present an extremely challenging situation for the friendly squad. We set up the enemy force this way to see if there were a set of factor levels that would consistently result in good performance, even in this unusually challenging situation. Figure 1 provides a diagram of this scenario.

As part of step 1 in defining the analysis, metrics are selected to help address the issues for analysis. With this generalized multiple RSM approach, performance metrics are identified and then prioritized based on the scenario and the issues for analysis. Although not required in all applications, a value function may be developed based on the prioritization of the individual responses. This value function may simply serve as a separate response in the concepts that follow. We use an approach described in 


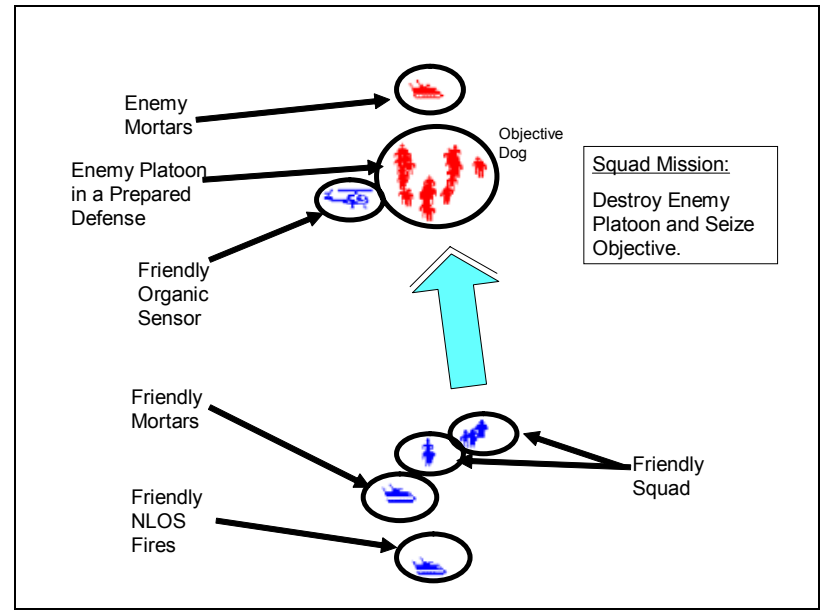

Figure 1: Squad Attack Scenario

(Ben-Gal and Bukchin 2002) that suggests the use of normalized performance measures to develop a "desirability function." The desirability function is given by:

$$
D F=\sum_{i=1}^{k} v_{i} z_{i}
$$

where $v_{i}$ is the importance value of the ith normalized response, $z_{i}$. The $v_{i}$ are developed so that $\sum_{i=1}^{k} v_{i}=1$ and $0 \geq v_{i} \geq 1$ for all $v_{i}$. Our approach is to conduct analysis based on the developed desirability function and each of the performance metrics individually, in sequence, based on the prioritization. Because we look at each of the performance metrics individually, this approach is robust to the selection of the $v_{i}$, (Brown and Schamburg 2004).

For our application, in part, the multi-response approach is related to the tactical mission described by the scenario. Our performance measures are directly related to the squad's survivability, the squad's lethality, seizing and controlling terrain, and friendly sensor survivability. In order of importance, the measures of performance include:

1. $y_{1}=$ (Lethality) the number of enemy casualties,

2. $y_{2}=$ (Survivability) the number of friendly casualties ,

3. $y_{3}=$ (Seizing the Objective) whether or not the squad seized the objective (zero if the squad does not seize the objective and one if the squad seizes the objective), and

4. $y_{4}=$ (Sensor Survivability) whether or not the friendly sensor is destroyed (zero if the sensor is not destroyed and one if the sensor is destroyed).
The prioritization of response values defined above helps to further define the squad mission. Therefore we call this desirability function the "mission response" function. For use with the mission response function, we normalize the responses in the following way.

1. Normalized Response Value for Lethality:

$$
z_{1}=\frac{\left(y_{1}-y_{1 \min }\right)}{\left(y_{1 \max }-y_{1 \min }\right)}
$$

2. Normalized Response Value for Survivability:

$$
z_{2}=\frac{\left(y_{2 \max }-y_{2}\right)}{\left(y_{2 \max }-y_{2 \min }\right)}
$$

3. Normalized Response Value for Seizing the Objective:

$$
z_{3}=y_{3}
$$

4. Normalized Response Value for Sensor Survivability:

$$
z_{4}=1-y_{4}
$$

Further defining the mission, enemy casualties are just as important as friendly casualties. The number of enemy casualties is just about twice as important as seizing the objective. Finally, seizing the objective is about twice as important as the friendly sensor's survivability. Given this information about the mission, the mission response value is further specified by the following equations. Based on the resulting responses, the mission response function is developed using (1) above. With this mission response function, larger is better, (Brown and Schamburg 2004).

$$
\begin{aligned}
& v_{1}=v_{2} \\
& v_{2}=2 v_{3} \\
& v_{3}=2 v_{4}
\end{aligned}
$$

Because of our techniques for using the responses individually, the analysis is robust to the selection of the importance values for the responses. Furthermore, if desired, the analyst can vary the response values and conduct subsequent iterations of the methodology. This would allow the analyst to see how sensitive the desirability function is to the selection of the response values.

In determining optimal solutions and constraining the decision space, step 7 of the methodology is accomplished by 
exploiting the special structure of outputs of the tree-based methods. CART's are used to determine near optimal settings and to find regions where good solutions exist. At the same time, CART is used to gain an initial understanding of the relationships and to help determine the next experimental region. Table 1 shows the general multiple response optimization approach through use of CART's. With CART, the analyst finds the best solutions through observation of the results at the terminal nodes. Then, he observes branches up the tree to define the regions in which those solutions lie. Using this special structure, we start with the regression tree for the desirability function in our application. Based on the prioritization of the other responses of interest, we repeat this process with the other trees, in order, until we have input related to all of the important variables in the study. In cases where we have conflicting input from the competing responses, preference is given to the trees related to the highest priority responses. Alternatively, judgment can be with-held until more analysis information is acquired. Robust solutions are desired. Therefore, we first use trees that are reduced to fewer terminal nodes (about 10 terminal nodes in our case). Terminal nodes that provide "good" solutions with many observations (say 500 or more in our case) may be preferred to terminal nodes that provide the "best" observed solution but have only a few observations (say 50 in our case). Using information this way, we constrain the decision space. Furthermore, we can constrain the experimental region for the next iteration of the generalized multiple RSM. In the final iteration(s), we use this information to determine near optimal solutions. The advantage of this method is that in early phases of the approach, the trees provide the analyst

Table 1: Multiple Response Optimization Process through use of CART's

Based on prioritization of the responses, near optimal settings and the constrained region are determined through an iterative sequence of analysis of the CART's.

\section{STEPS}

a) Use smaller pruned trees so that terminal nodes have large number of observations.

b) Start with tree for most important response.

c) Develop constraints based on one or two "good" terminal nodes.

d) Where conflicts exist, with-hold judgment on that variable value or develop constraint based on higher priority tree.

e) Go to tree for the next most important response and go to step c.

f) If more information is needed about any variable(s), consider using larger trees and go back to step $b$. with information about the entire decision space. Constraining experimental regions in this way, the analyst can continue with iterations of the methodology until a near optimal solution is found or until there is relatively little change in the response(s), (Schamburg 2004).

As a potential alternative approach, the CART optimal solution can then be used to reduce the size of the developed empirical models. It may be especially helpful in reducing the combinatorial nature and size of the solution space in the models with many RCS terms. Here, the analyst can "fathom" many of the branches of the model with RCS terms by reducing the range of variable values. In agreement with the knot locations, some of the RCS terms can be dropped from the model and others can be treated as normal cubed terms. For accurate empirical models, further improvement might be beneficial. Other methods involve using the CART solution as a good start point for optimization of the empirical model. From the start point, one can use heuristic search techniques or mathematical programming methods on the empirical model in an effort to find "better" solutions.

For comparison, the fitted model found as a result of TRSM techniques is typically useful for only a small portion of the entire operability region. Optimization of the model, given the range of the variable values used in the experimentation, leads to selection of a new experimental region in which case this methodology is iterated. The analysis uses gradient information from the developed models and moves in the direction of steepest descent. Alternatively, optimization of the model may result in finding a stationary point or a local optima constrained by the boundaries of the operability region. The RSM models may be optimized by using linear or nonlinear programming software, depending on the form of the function.

One beneficial side-effect of the RSM approach for linear optimization is that mathematical programming techniques allow for methods of sensitivity analysis, including evaluation of reduced costs, slack (or surplus) values, and dual variables. For a minimization problem, the reduced cost is the predicted increase in the objective function value if there is a small, feasible change in the associated variable. Therefore, in an optimal solution (for a minimization problem), all reduced costs should be greater than or equal to zero. The slack (or surplus) values show which constraints are binding and which are not. Non zero slack values show how much of a given resource is available in the optimal solution. Binding constraints have a slack value of zero. The dual variables describe the potential rate of improvement in the objective function value if the corresponding constraint is slightly relaxed. Only binding constraints will have non zero dual variables.

Because of these analysis advantages, in some analysis cases, it may be beneficial to revert to the TRSM approach in the final iteration(s) of this generalized multiple RSM. In other words, once the generalized multiple RSM decision space is constrained to a small region through use of 
the described CART techniques, one can use the common TRSM experimental designs and modeling techniques for the final iterations of the analysis.

In our application, Figure 2 shows the initial reduced regression tree for the mission response function, (Brown and Schamburg 2004). The input variables in our analysis are abbreviated as four and five letter combinations at the branches of the tree. In our actual application, 44 different input variables were considered. The best average solution of .7242 is found at the terminal node where the variable STAWW $>29$. From this terminal node, working back through the branches of the tree, this average solution is found when SLMSR $>147$, STMDE $>1$, SMSR $>249$, SLAFW $>-57$, STMSR $>64$, and STAWW $>29$. While using CART this way, we must be cautious about decisions involving final factor values. During this process other branches should be evaluated as well. For example, a second look at Figure 2 reveals that a second best solution of .7193 is found. At this node, SLMSR $>147$, but STMDE $<1$. This information is conflicting with the previous solution found. After analysis of the larger mission response regression tree, good solutions are found when STMDE $<$ 1 and when STMDE $>1$. Therefore, we with-hold judgment about the best STMDE branch and note that STMDE may have important interactions with the other variables identified in these solutions. Note that when STMDE $<1$, that it is better for SLAFW $<-66.5$. This is generally consistent with the first solution identified, (Brown and Schamburg 2004).

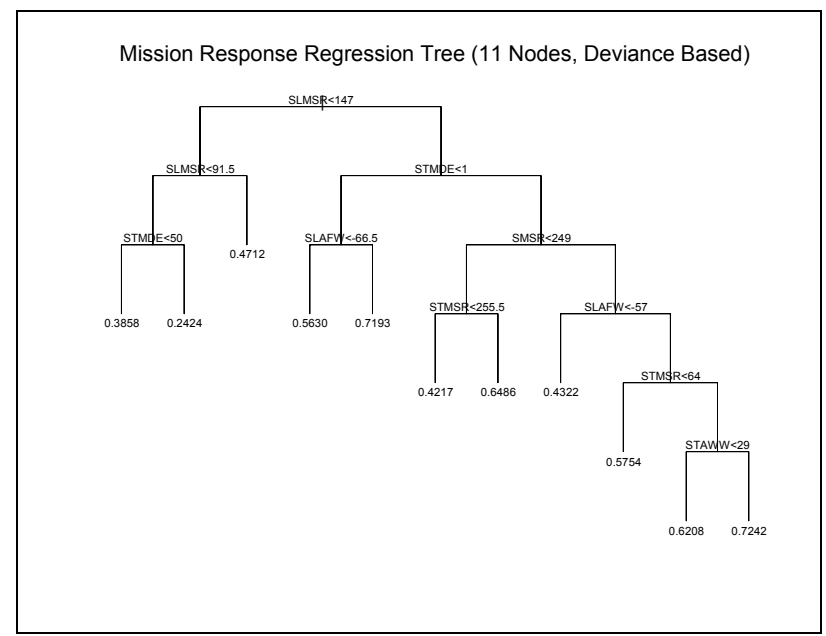

Figure 2: Regression Tree for the Mission Response Function, (Brown and Schamburg 2004)

The information from the tree in Figure 2 plus information from CART analysis based on the other individual responses is used to gain a better understanding of the variable relationships. It is also used to help select subsequent experimental regions. Table 2 provides a partial summary of the initial findings through use of CART. To develop this summary, we started with reduced trees for each of the re- sponses (similar to Figure 2). To gain more information, we expanded the analysis and used larger trees as appropriate.

Table 2: Part of Summary of CART Findings for First Iteration of the Analysis

\begin{tabular}{|l|l|l|l|l|l|l|}
\hline Variable & $\begin{array}{l}\text { MSN } \\
\text { Re- } \\
\text { sponse } \\
\text { Fun }\end{array}$ & $\begin{array}{l}\text { Blue } \\
\text { Cas }\end{array}$ & $\begin{array}{l}\text { Red } \\
\text { Cas }\end{array}$ & $\begin{array}{l}\text { Seize } \\
\text { Obj }\end{array}$ & $\begin{array}{l}\text { Sensor } \\
\text { Sur- } \\
\text { vive }\end{array}$ & $\begin{array}{l}\text { Over- } \\
\text { all }\end{array}$ \\
\hline SLMSR & $>147$ & $>147$ & $>124$ & $>179$ & & $>147$ \\
\hline STAWW & $>29$ & & $>-1$ & $>-1$ & & $>-1$ \\
\hline SMSR & $>249$ & $>14$ & $>165$ & $>184$ & $<494$ & $>165$ \\
\hline SLAFW & $>-67$ & $>-47$ & $>-68$ & $>-39$ & & $>-67$ \\
\hline STMSR & $>64$ & $>73$ & $>91.5$ & $>128$ & & $>64$ \\
\hline SLAEW & $>-70$ & & $>6$ & $>-21$ & & $>-70$ \\
\hline ATMDE & $<32$ & & & $<179$ & & $<32$ \\
\hline STMDE & $<1$ & & $<157$ & & & $<1$ \\
\hline SLCD & & & & $<113$ & & \\
\hline ATAEW & & $<-1$ & & $>-29$ & & $<-1$ \\
\hline STAEW & & $>-5$ & & $>33.5$ & & $>-5$ \\
\hline ATMSR & & $>69$ & & $<294$ & & $>69$ \\
\hline
\end{tabular}

Table 3 shows the mean performance of the entire initial dataset and six example solutions that were developed through use of our techniques. These solutions show a marked increase in performance considering the average results of the complete data set. This is especially true when considering the challenging scenario that was developed for this analysis. Solutions 1 through 4 were developed based on one iteration of the methodology. Solutions 5 and 6 were developed based on a second iteration of the methodology. The solution performance comparison is based on 50 observations of each solution. Almost perfect performance was obtained by all solutions for the seize the objective response and the sensor survivability response. Through the use of box plots, Figure 3 shows the distribu-

Table 3: Mean Performance for the Entire Experimental Region Data Set and the Best Solutions Found

\begin{tabular}{|l|r|r|r|r|r|r|r|}
\hline & \multicolumn{7}{|c|}{ Solution Number } \\
\hline & $\begin{array}{l}\text { Complete } \\
\text { Data Set }\end{array}$ & 1 & \multicolumn{1}{|c|}{2} & \multicolumn{1}{|c|}{3} & 4 & 5 & 6 \\
\hline $\begin{array}{l}\text { Blue Kills } \\
\text { (Survivability) }\end{array}$ & 3.7 & 1.14 & 0.02 & 0.68 & 1.74 & 0.94 & 0.24 \\
\hline $\begin{array}{l}\text { Red Kills } \\
\text { (Lethality) }\end{array}$ & 26.9 & 34.3 & 33.3 & 37.5 & 36.4 & 37.2 & 37.6 \\
\hline $\begin{array}{l}\text { Seize Objective } \\
\text { (Yes=1) }\end{array}$ & 0.56 & 0.98 & 1 & 1 & 0.96 & 1 & 1 \\
\hline $\begin{array}{l}\text { Sensor De- } \\
\text { stroyed (Yes=1) }\end{array}$ & 0.71 & 0.02 & 0 & 0.06 & 0.06 & 0 & 0 \\
\hline $\begin{array}{l}\text { Mission Re- } \\
\text { sponse Value }\end{array}$ & 0.59 & 0.90 & 0.95 & 0.95 & 0.89 & 0.95 & 0.98 \\
\hline
\end{tabular}




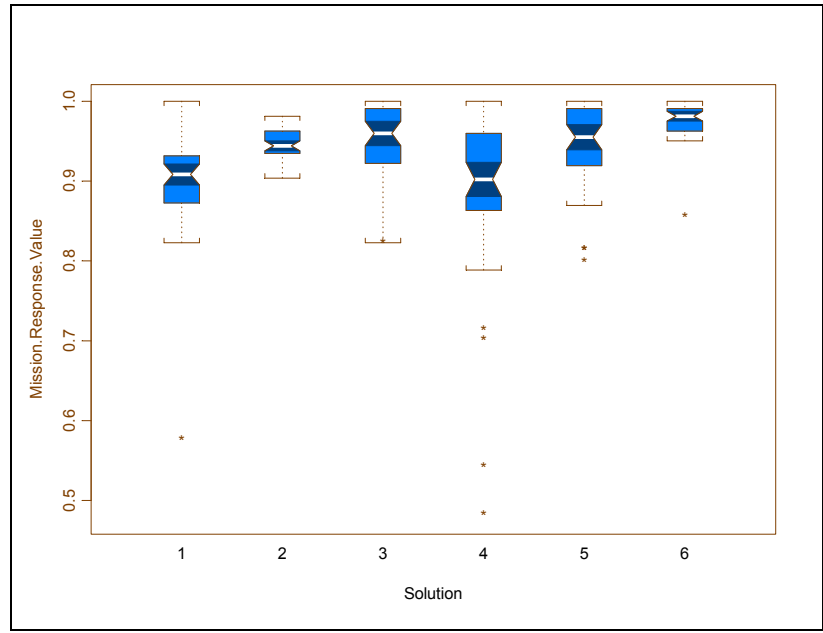

Figure 3: Box plots for Comparison of the Distributions of Solution Performance Based on Mission Response (Each Graph Represents 50 Observations of Each Solution)

tions of these observations for the mission response, (Brown and Schamburg 2004).

For the mission response, solution 6 resulted in the best performance. Solutions 3 and 5 performed well but had some observations that were below .90 . Solution 2 has a tighter distribution but does not have many observations that are above .95. Using the nonparametric KruskalWallis test to compare the differences of these distributions for the mission response value, we found that there was a significant statistical difference between at least two of the distributions at the .00 significance level. When comparing the distribution of solution 6 to the other solutions individually, there was a significant statistical difference at the .01 significance level. The Chi-square statistic was the smallest (at 10.8) when comparing solution 6 to solution 5 .

Through use of box plots and statistical tests, we also conducted comparisons based on performance with respect to the other four responses. Overall, solution 6 resulted in the best performance. In terms of the mission response function and red casualties, there was a significant statistical difference between solution 6 and the other solutions individually. However, solution 2 performed the best in terms of survivability. Solution 6 was found during the $2^{\text {nd }}$ iteration of the methodology. However, it was developed by reviewing concepts that were discovered earlier in the analysis. Furthermore, in solution 6 , the technological related factors are not at their maximum allowable levels.

\section{SUMMARY AND CONCLUSIONS}

In summary, our application of the generalized multiple RSM involves the following techniques:

1. Conduct an iterative sequence of Latin hypercube experimental designs, analysis of CART \& function approximations w/ RCS terms.
2. Begin with a large set of experiments and conduct "search" analysis iteration to gain an understanding of the broader decision space and to determine the analysis area(s) of interest.

3. Based on prioritization of responses, use CART to gain an understanding of the variable relationships and to constrain the decision space. Use higher order models to help further understand important factors and relationships.

4. Conduct experiments and analysis in sequentially constrained decision space until near optimal solutions are found or until change in the response(s) is minimal.

The generalized multiple RSM provides an approach for analysis of simulation optimization problems involving many factors and multiple responses of interest. Initially, the focus of the analysis is developed so that the investigation is pointed at answering the important issues. Through use of the methodology, one gains an understanding of a large decision space and factor levels that lead to good solutions. This results in generalizations that can ultimately be used to provide input to decision making or follow-on analysis requirements.

The described generalized multiple RSM is intended to provide a general approach to analysis involving complex systems simulations. This analysis process is intended to be iterative. The iterative approach helps verify the conclusions drawn from previous phases of the process. It additionally leads to increased understanding of the complex relationships involved in large systems. The study should be set up so that one may gain information from the analysis of a given scenario that may be beneficial in the analysis of upcoming scenarios.

The methodology is also intended to be flexible. The steps and tools described above should be adapted to the problem and the issues at hand. While the TRSM provides a useful approach to more specific analysis situations involving fewer factors, this generalized approach broadens TRSM concepts by incorporating other data mining techniques. The use of Latin hypercube designs provides enough information about the broad solution space to make this possible. The use of CART and higher order function modeling techniques were selected for ease of interpretation and for the natural way they allow one to gain increased understanding over the experimental regions of interest.

Through the use of CART's to define regions of importance and regions for subsequent iterations of analysis, the generalized multiple RSM provides a robust way to deal with multi-response analysis problems. The method requires prioritization of the response measures of interest. Then the analyst can make decisions about regions of interest based on information from a series of trees that address each of the responses. This process makes the tradeoffs among the responses clear. 


\section{REFERENCES}

Ben-Gal, I., and J. Bukchin. 2002. The Ergonomic Design of Workstations using Virtual Manufacturing and Response Surface Methodology. IIE Transactions 34: 375 to 391.

Box, G. E. P., and N. R. Draper. 1987. Empirical ModelBuilding and Response Surfaces. New York: John Wiley and Sons.

Brown, D. E., and J. B. Schamburg. 2004. A Modified Response Surface Methodology for Knowledge Discovery with Simulations. Submitted to European Journal of Operations Research (Elsevier).

Cioppa, T. M. 2002. Efficient Nearly Orthogonal and Space-Filling Experimental Designs for HighDimensional Complex Models. Dissertation, Naval Postgraduate School, Monterey, CA.

Harrell, F.E. 2001. Regression Modeling Strategies. New York: Springer-Verlag Inc.

Schamburg, J. B. 1995. Deployment Planning and Analysis for Time Difference of Arrival and Differential Doppler Location Finding Assets. Thesis, University of Virginia, Charlottesville, Virginia.

Schamburg, J. B. 2004. A Modified Response Surface Methodology for Knowledge Discovery. Dissertation, University of Virginia, Charlottesville, Virginia.

Stone, C. J., and C. Y. Koo. 1985. Additive Splines in Statistics. Proceedings of the Statistical Computing Section ASA: 45-48.

\section{AUTHOR BIOGRAPHIES}

DONALD E. BROWN, is Professor and Chair of the Department of Systems and Information Engineering, University of Virginia. He is a Fellow of the IEEE and the Editorin-Chief of the IEEE Transactions on Systems, Man, and Cybernetics, Part A: Systems and Humans. He is the recipient of the IEEE Norbert Wiener Award and the IEEE Millennium medal. Dr. Brown received a Ph.D. from the University of Michigan in Industrial and Operations Engineering. He does research in data fusion, predictive modeling, and response surfaces with applications to security and safety.

JEFFREY B. SCHAMBURG, is an Assistant Professor and an Operations Research Analyst in the Department of Systems and Information Engineering and the Operations Research Center at West Point, NY. Lieutenant Colonel Schamburg received his commission as an Infantry Officer and a B.S. in Civil Engineering from the United States Military Academy, West Point in 1986. He received his Ph.D. in Systems and Information Engineering from the University of Virginia in 2004. His present research interests include data mining, predictive modeling, and response surface methodology with applications to military, information, and industrial systems. 